\title{
New Opportunities for Automated Pedestrian Performance Measures
}

\author{
Jack Bell, Corresponding Author \\ Purdue University \\ 207 S Martin Jischke Dr., West Lafayette, IN 47907 \\ Phone: 765-494-6508 \\ Email: bell224@purdue.edu

\section{Rik Law} \\ Purdue University \\ 207 S Martin Jischke Dr., West Lafayette, IN 47907 \\ Phone: 484-885-9679 \\ Email: law14@purdue.edu

\section{Howell Li} \\ Purdue University \\ 207 S Martin Jischke Dr., West Lafayette, IN 47907 \\ Phone: 765-494-9601 \\ Email: howell-li@purdue.edu
}

\section{Ben Anderson}

City of West Lafayette

705 S River Rd., West Lafayette, IN 47906

Phone: 765-404-8441

Email: banderson@westlafayette.in.gov

\section{Darcy M. Bullock}

Purdue University

207 S Martin Jischke Dr., West Lafayette, IN 47907

Phone: 765-496-2226

Email: darcy@purdue.edu

August 1, 2019

\footnotetext{
${ }^{1}$ Submitted to the Transportation Research Board (TRB) 99th annual meeting on August 1, 2019. Presented at the 106th Purdue Road School on March 10, 2020.
} 


\section{ABSTRACT}

Pedestrian safety is an important concern when evaluating intersections. Previous literature has shown that exclusive pedestrian phases improve safety, but at the expense of imposing greater pedestrian and motorist delay. However, outside of crash data, there are no easily implementable performance measures for pedestrians at traffic signals. This study proposes two performance metrics: (1) a time-to-jaywalk measure, and (2) the Conflict Occupancy Ratio (COR) for evaluating concurrent pedestrian signal phasing with turning vehicles. The COR quantifies conflicts between turning vehicles and pedestrians in the crosswalk. The COR is based upon a commercially deployed video detection system that correctly identified the presence of pedestrians to within two per cycle in this study. This performance is likely sufficient for the current application, but as the technology matures it will provide a scalable screening tool to identify intersections that have opportunities for capacity adjustments or warrant further direct field investigation.

Keywords: pedestrian safety, vehicle conflicts, exclusive phase, occupancy ratio, video detection, automated traffic signal performance measures 


\section{Table of Contents}

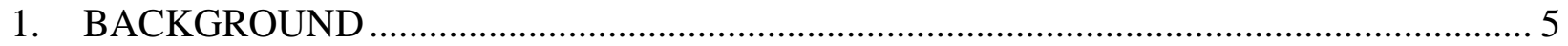

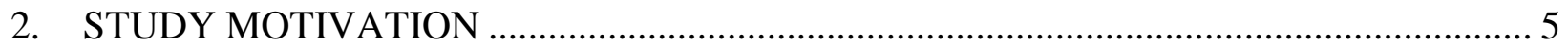

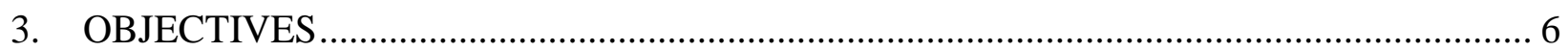

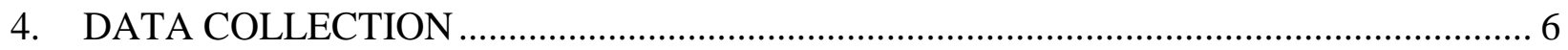

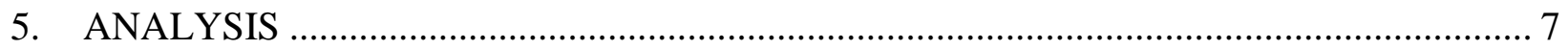

5.1 Groundtruth of Video Detection Algorithm …………..................................................... 7

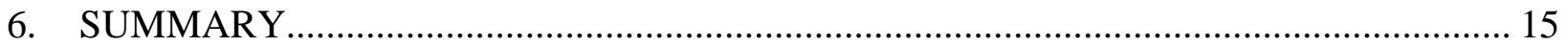

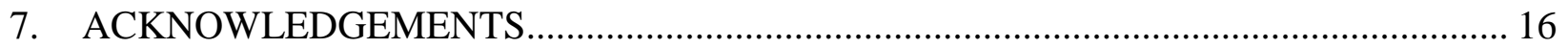

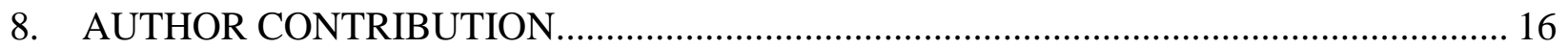

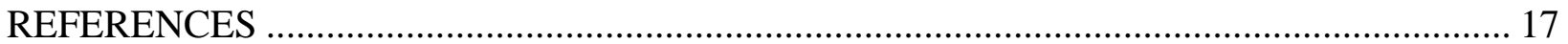




\section{List of Figures}

Figure 4.1 Data collection locations (maps and images from google maps) .............................. 7

Figure 5.1 Pedestrians identified by algorithm versus actual (manual) count ............................. 8

Figure 5.2 Grant Street Intersection: Pedestrians waiting, two cycles .................................... 9

Figure 5.3 Grant Street intersection: Time to jaywalk........................................................ 11

Figure 5.4 Chauncey Avenue: Occupancy of vehicles and pedestrians relative to the signal

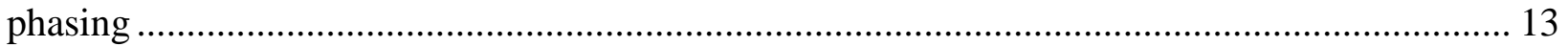

Figure 5.5 Chauncey Avenue: Pedestrian and vehicle occupancy sorted by COR .................... 14

\section{List of Tables}

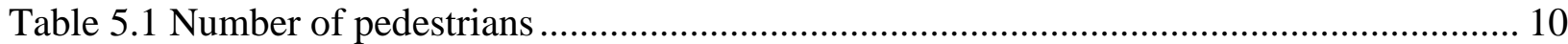

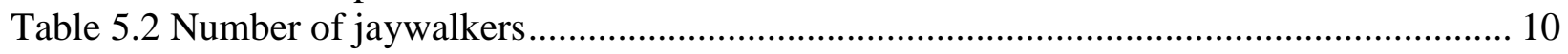

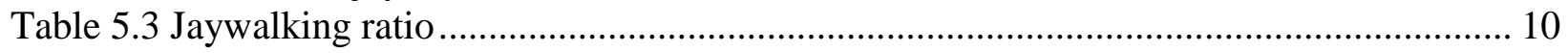




\section{BACKGROUND}

Historically, the term jaywalking has been used almost as long as automobiles have existed from the early 1900s. Jouneau and Carincotte define jaywalking as when pedestrians cross the intersection or street without respecting the crosswalk, or when using the crosswalk during the pedestrian DON'T WALK phase (1). Jaywalking events are very common at intersections and can affect the safety and operations of a traffic signal. In 2017, 16.1\% of total fatal crashes involved pedestrians (2). Jaywalking events are highly concentrated and can be influenced by the crossing environment, such as pedestrian and vehicular volume, bus stops presence, and crossing distance (3). A study by the Federal Highway Administration indicates that jaywalking is affected by the distance between marked crosswalks, annual average daily traffic (AADT), physical barriers that might prevent pedestrians from easily crossing the roadway, the presence and location of bus stops, the number of potential pedestrian trip originators and destinations, the presence of a "right turn only" lane, the width of the roadway/pedestrian crossing, and the presence of a T-intersection between the two marked crossings (4). Several studies evaluated people's intentions to cross the road and the psychological factors in which they choose to do so $(5,6)$.

Many intersections in the United States use concurrent phasing for pedestrian traffic. It is defined by PEDSAFE (7) as when the pedestrian WALK turns on concurrently with the parallel vehicle phases allowing motorists to turn either left or right through the crosswalk after yielding. Studies have found that permitting vehicles to travel through the same path and the same time as pedestrians can lead to more conflicts between pedestrian and vehicular traffic (8). These conflicts have been modeled in the past using vehicle presence detectors in right turning lanes and parallel crosswalks to quantify the occupancy of vehicles and pedestrian delay (9). Simulations have also been created based on majority game theory (10). Additionally models have also been created using pedestrian and vehicular flow rates to calculate the likelihood of conflicts $(11,12)$.

\section{STUDY MOTIVATION}

Recent developments in high-resolution video detection systems and intelligent software algorithms have enabled new ways of capturing position and movements of individual pedestrians, bicyclists, and other types of micro-mobility at an intersection (13). Traditionally, pedestrian detection consists of registering the time and phase of an associated button push at a traffic signal $(14,15)$. With the new technology there is an opportunity to leverage the algorithms to identify movements with a high number of conflicts involving vehicles and pedestrians at different times of day and at different locations. Shiravi et al. explored using computer vision to quantify pedestrian and crosswalk performance in terms of occupancy, clearance time, compliance, and exposure by pedestrian phase status (13). There is opportunity to look at jaywalking as a function of delay from the onset of solid DON'T WALK and conflicts at parallel crosswalks with turning traffic. 


\section{OBJECTIVES}

The objectives of this study are (1) to assess the performance of video pedestrian detection technology; (2) to build upon existing research and develop a performance metric for evaluating jaywalking at an intersection; (3) to develop a pedestrian-vehicle conflict metric that can be applied by video detection; and (4) assess performance of the developed metrics at an intersection.

\section{DATA COLLECTION}

During this study, data on pedestrians and vehicles was collected at two different intersections in West Lafayette, Indiana. One is an exclusive pedestrian phase at State Street and Grant Street and the second is a concurrent pedestrian phase at State Street and Chauncey Avenue. The two intersections are shown in Error! Reference source not found.. These State Street intersections are at Grant Street (callout i in Figure 4.1a) and Chauncey Avenue (callout ii in Figure 4.1a) are instrumented with multimodal video detection systems. The Grant Street intersection (Figure 4.1b) uses an exclusive pedestrian phase of 30 seconds with a cycle length ranging from 65 to 210 seconds, and the Chauncey Avenue intersection (Figure 4.1c) uses concurrent pedestrian phasing with a split of 70 to more than 300 seconds. The video detection system has the ability to classify and track the movements of all vehicles and pedestrians. Video is continuously recorded for all traffic movements daily in 15-minute archives. Data collection is performed by watching these recordings for a number of cycles during peak and non-peak periods for each intersection.

At Grant Street, the exclusive pedestrian phase is served after the vehicular phases. Data was collected for two periods (peak, non-peak) each day over the two weeks of 6/11-6/24. Peak is considered between $12 \mathrm{pm}-1 \mathrm{pm}$ and non-peak is between $6 \mathrm{pm}-7 \mathrm{pm}$. Each corner is labelled by direction: northwest (NW), northeast (NE), southwest (SW), southeast (SE), and a designated area for waiting. The total number of waiting pedestrians is counted in one-second bins at each corner for two consecutive cycles per analysis. Jaywalkers crossing outside of the waiting zone are counted on the corresponding corner as both entering and exiting the zone in the same interval of time. Pedestrians arriving and departing during the exclusive pedestrians phase are counted as both entering and exiting the zone in the same interval of time.

At Chauncey Avenue, the focus is on the north-side crosswalk parallel to State Street (callout iii in Figure 4.1c). It was chosen because the crosswalk lies alongside a curb-separated two-way cycle track. The presence of the cycle track renders the conflict zone to be larger than intersections without this facility. The conflict zone used in this study at this intersection spans the entirety of the crosswalk and path between the east-side crosswalk and the north-side crosswalk that right turning vehicles would follow.

Intersection occupancy for vehicles is measured from the time that a right-turning vehicle's front bumper is past the east-side crosswalk, until its rear bumper is past the north-side crosswalk. Crosswalk occupancy for pedestrians is measured from the time a pedestrian enters the crosswalk until the time all pedestrians exit the crosswalk. Jaywalkers crossing well outside of crosswalk area are not included in the occupancy count. 


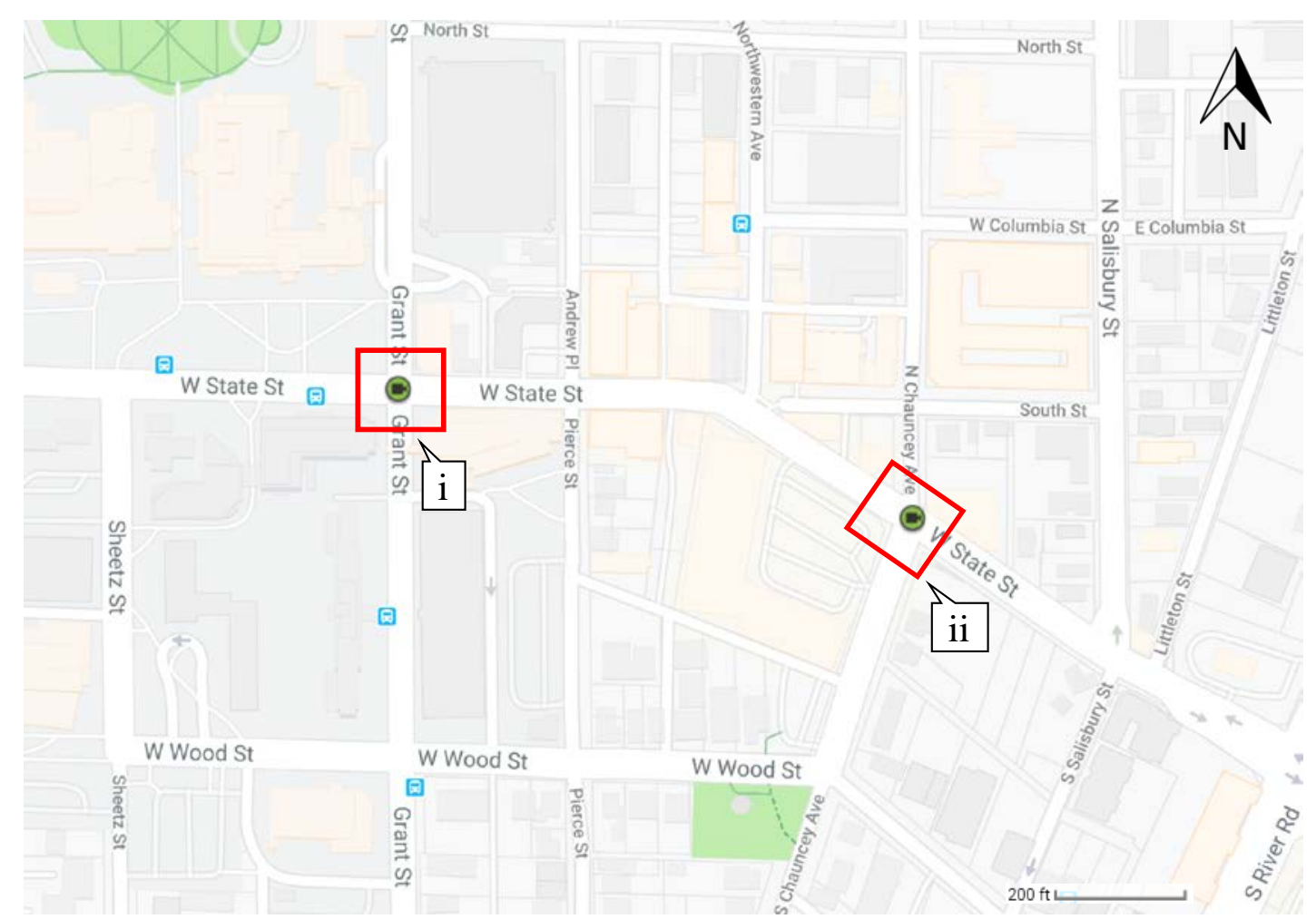

(a) View of both study intersections

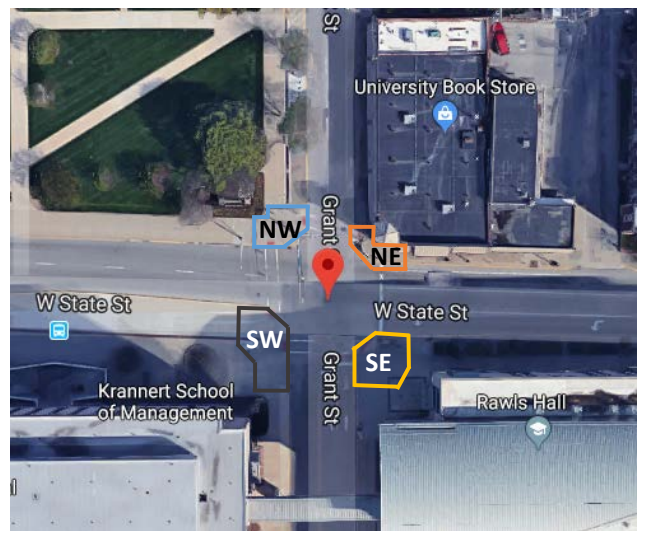

(b) Grant and State intersection

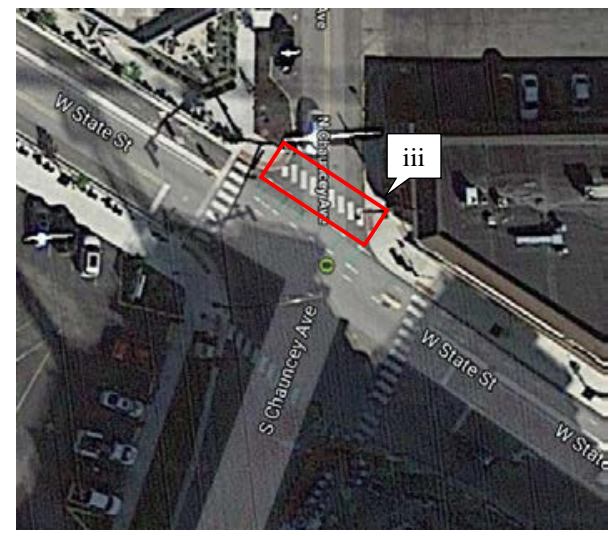

(c) Chauncey and State intersection

Figure 4.1 Data collection locations (maps and images from google maps).

\section{ANALYSIS}

\subsection{Groundtruth of Video Detection Algorithm}

An analysis of the pedestrian detection algorithm is conducted for 32 consecutive cycles at the Chauncey and State intersection. The number of boxes that appeared on the video display shown as "ped" are counted and labelled per cycle, and also counted manually (Figure 5.1). The counts derived from the automated system are labeled "algorithm," and the number of pedestrians that appeared near the crosswalk are counted manually and labelled “actual” (Figure 5.1a). 
The algorithm is accurate within two pedestrians per cycle, which agrees with the product specification for low-volume movements (16). When comparing these numbers cumulatively (Figure 5.1b), the algorithm counted 27\% more pedestrians than manual counting (Figure 5.1b, callout $\mathrm{i}$ ). The accuracy is adequate for tabulating pedestrian-oriented performance measures to be used for screening for further study and analysis.

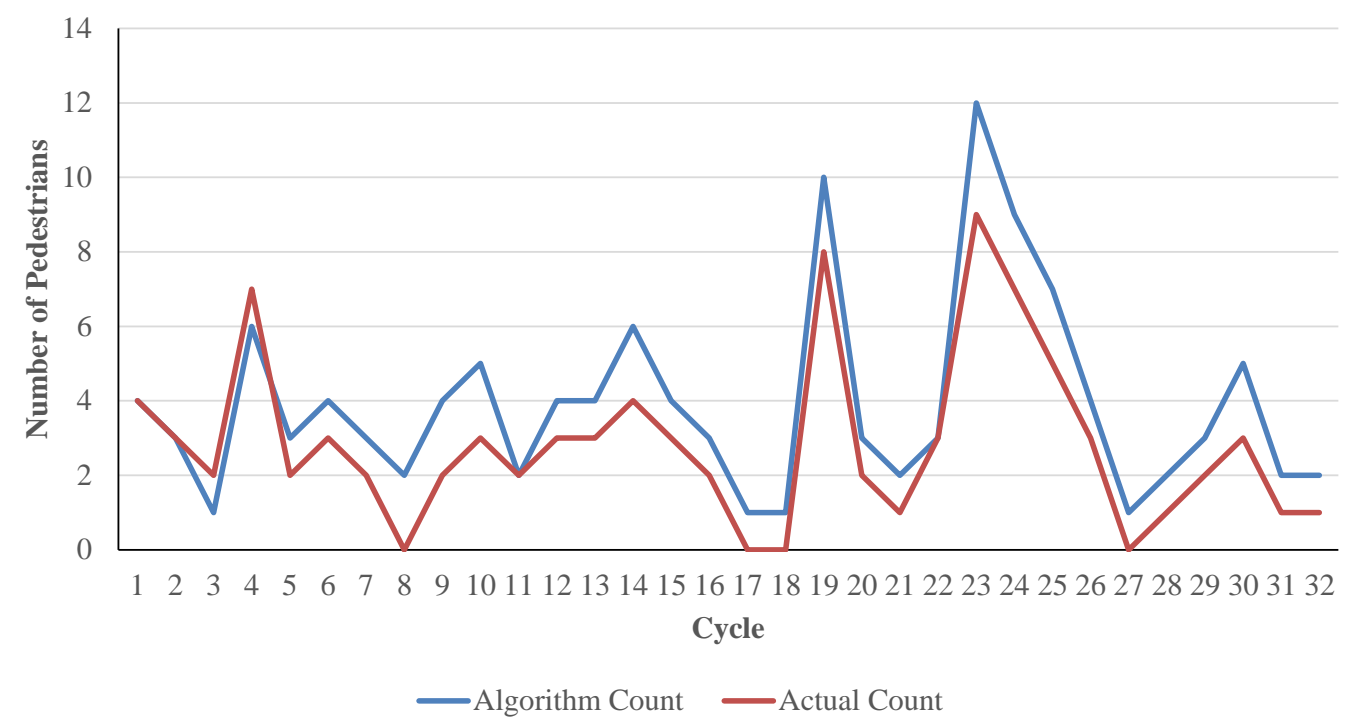

(a) Consecutive cycle

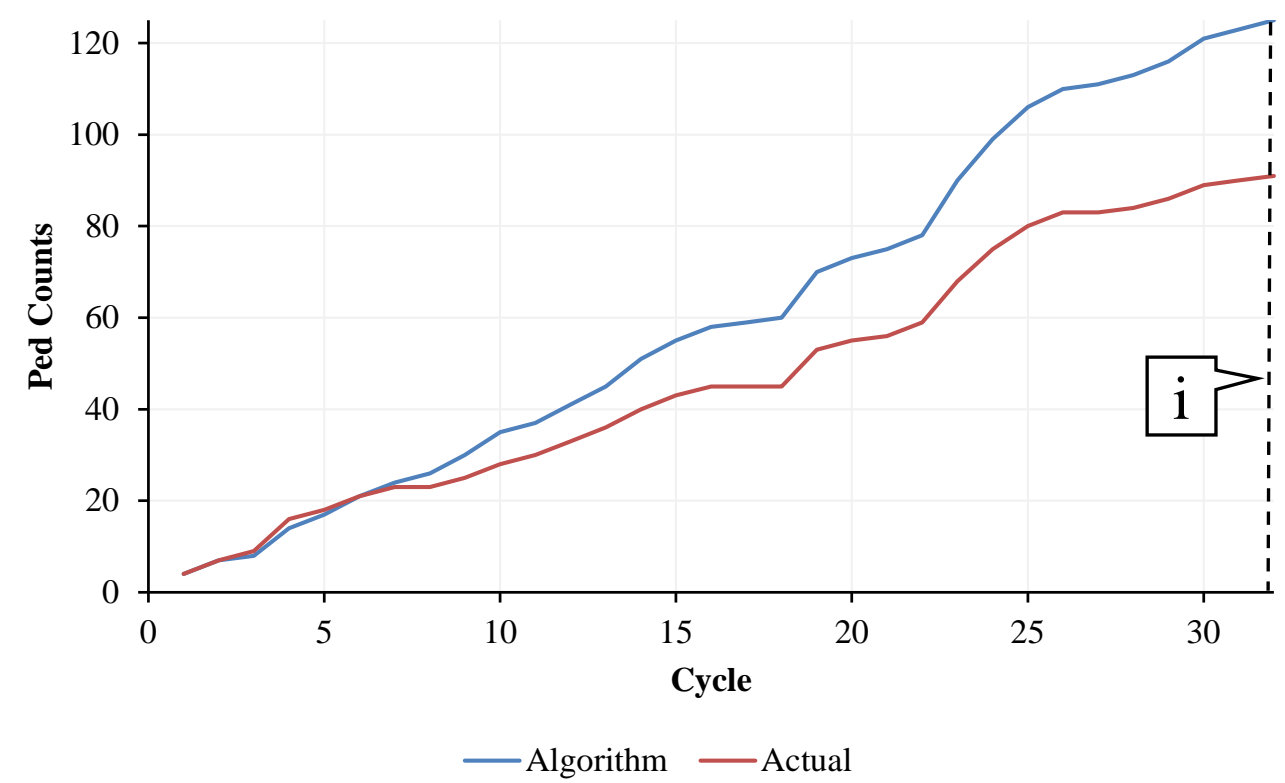

(b) Cumulative

Figure 5.1 Pedestrians identified by algorithm versus actual (manual) count. 


\subsection{Grant Street Jaywalking}

The study covered 28 peak cycles and 28 non-peak cycles for 14 days to determine the number of jaywalkers at Grant Street. Out of 819 pedestrians, 89 jaywalked over the two week sample. Figure 5.2 illustrates the number of pedestrians waiting at the intersection relative to the signal phasing for two cycles. In Figure 5.2a, the number of waiting pedestrians are accumulated in the stacked area graph colorized by the corner of the intersection where the pedestrians are waiting. The phasing for the vehicular movements, combined for both left turn and through phases of each cross street, are summarized in the red and green bar graphs for the same time period.

In Figure 5.2a, the red circles on the graph show cases where a pedestrian jaywalked. Callout i shows a pedestrian that has stepped off the curb at the NW corner in the first cycle, reducing the total number of pedestrians by one and footage is shown in Figure 5.2b. Callout ii and callout iii shows two additional pedestrians jaywalking in the second cycle.
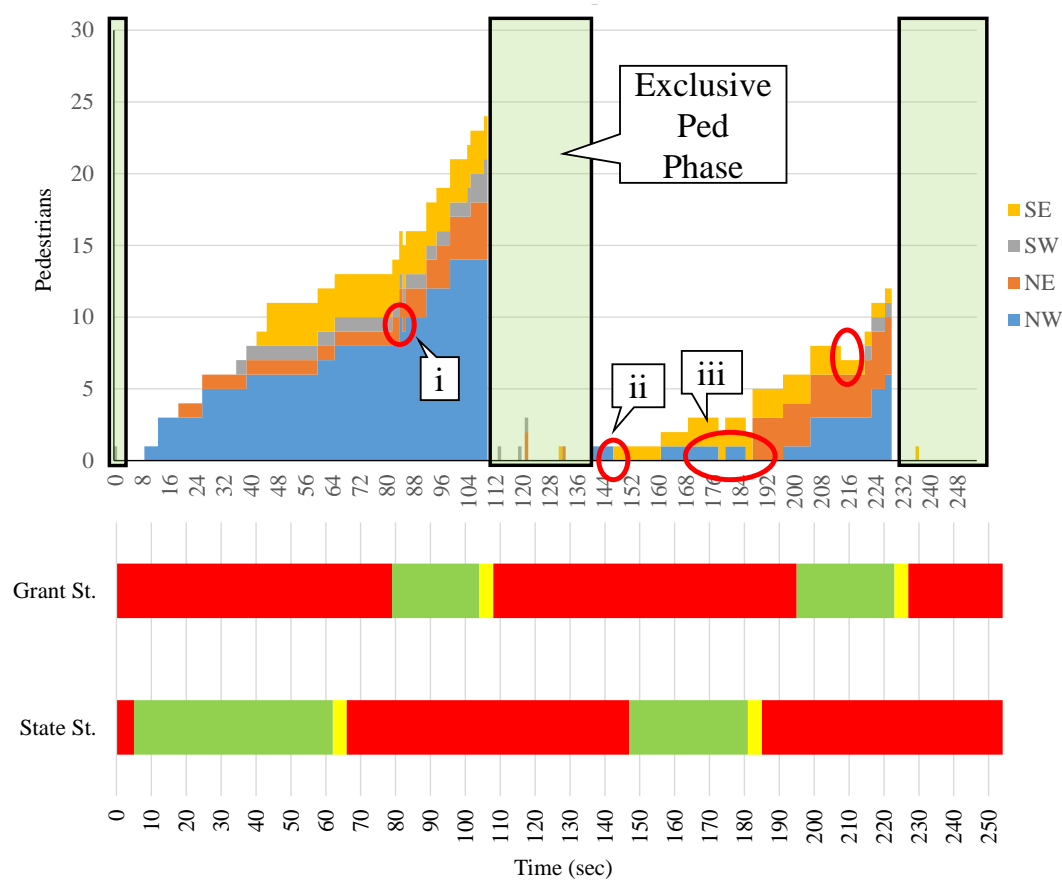

(a) Number of waiting pedestrians at Grant Street

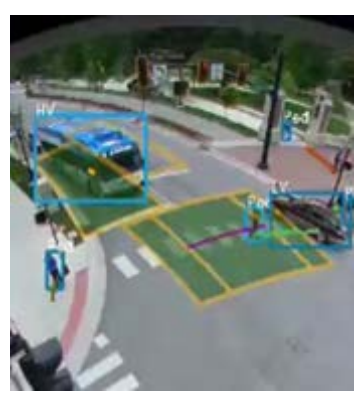

Callout i

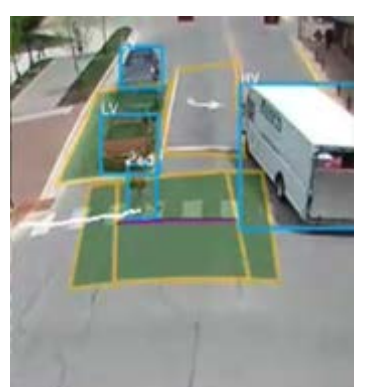

Callout ii

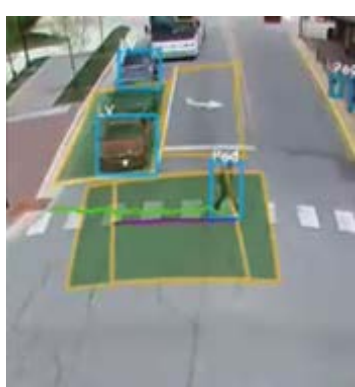

(d) Callout iii

Figure 5.2 Grant Street Intersection: Pedestrians waiting, two cycles. 
The tables below summarize the two weeks of data for the Grant Street intersection. Table 5.1 represents the number of pedestrians per day of the week. Grant Street saw the most foot traffic during the peak hours on weekdays from $12 \mathrm{pm}-1 \mathrm{pm}$. Table 5.2 highlights the number of jaywalkers during each day. Table 5.3 depicts the ratio of jaywalking to pedestrians for each day over two weeks. Pedestrians jaywalked more during the weekend. The data shows that the number of jaywalkers are 4 percentage points higher during non-peak times. Using the time between arrival and jaywalk, the median dwell time for a jaywalking pedestrian before stepping off the curb during peak hours is 16 seconds and the median wait time during non-peak hours is 8 seconds. Pedestrians wait longer before jaywalking during the peak hours and a greater percentage of pedestrians jaywalk during non-peak hours.

Table 5.1 Number of pedestrians

\begin{tabular}{l|ccccccc|c}
\multicolumn{1}{l}{} & MON & TUES & WED & THURS & FRI & SAT & SUN & \\
\hline PEAK & 84 & 114 & 89 & 109 & 120 & 21 & 21 & 558 \\
NONPEAK & 50 & 29 & 50 & 49 & 33 & 36 & 14 & 261 \\
\hline & 134 & 143 & 139 & 158 & 153 & 57 & 35 & 819
\end{tabular}

Table 5.2 Number of jaywalkers

\begin{tabular}{l|ccccccc|c}
\multicolumn{1}{l}{} & MON & TUES & WED & THURS & FRI & SAT & SUN & \\
\hline PEAK & 1 & 6 & 2 & 22 & 8 & 6 & 8 & 53 \\
NONPEAK & 8 & 4 & 5 & 6 & 2 & 3 & 8 & 36 \\
\hline & 9 & 10 & 7 & 28 & 10 & 9 & 16 & 89
\end{tabular}

\section{Table 5.3 Jaywalking ratio}

\begin{tabular}{l|ccccccc|c}
\multicolumn{1}{l}{} & MON & TUES & WED & THURS & FRI & SAT & SUN & \\
\hline PEAK & 0.01 & 0.05 & 0.02 & 0.20 & 0.07 & 0.29 & 0.38 & 0.10 \\
NONPEAK & 0.16 & 0.14 & 0.10 & 0.12 & 0.06 & 0.08 & 0.57 & 0.14 \\
\hline & 0.07 & 0.07 & 0.05 & 0.18 & 0.07 & 0.16 & 0.46 & 0.11
\end{tabular}

Jaywalking can cause unexpected pedestrian-vehicle interactions to occur. Over the analysis period, 63 pedestrians jaywalked parallel to the thru movement being served while 26 pedestrians walked orthogonal to the thru movement. In addition, 4 pedestrians jaywalked outside of the intersection (e.g., crossing upstream or mid-block). Jaywalkers crossing parallel to moving traffic can still obstruct the left or right turning movements.

The study proposes a time-to-jaywalk metric that identifies the amount of time after the beginning of the solid DON'T WALK indication by tracking the pedestrian when he/she steps off the curb. The performance measure is presented as a column chart in Figure 5.3. Overall, for the peak period (Figure 5.3a) the median time-to-jaywalk is 42 seconds. The median value for the non-peak period (Figure 5.3b) is 36 seconds. 


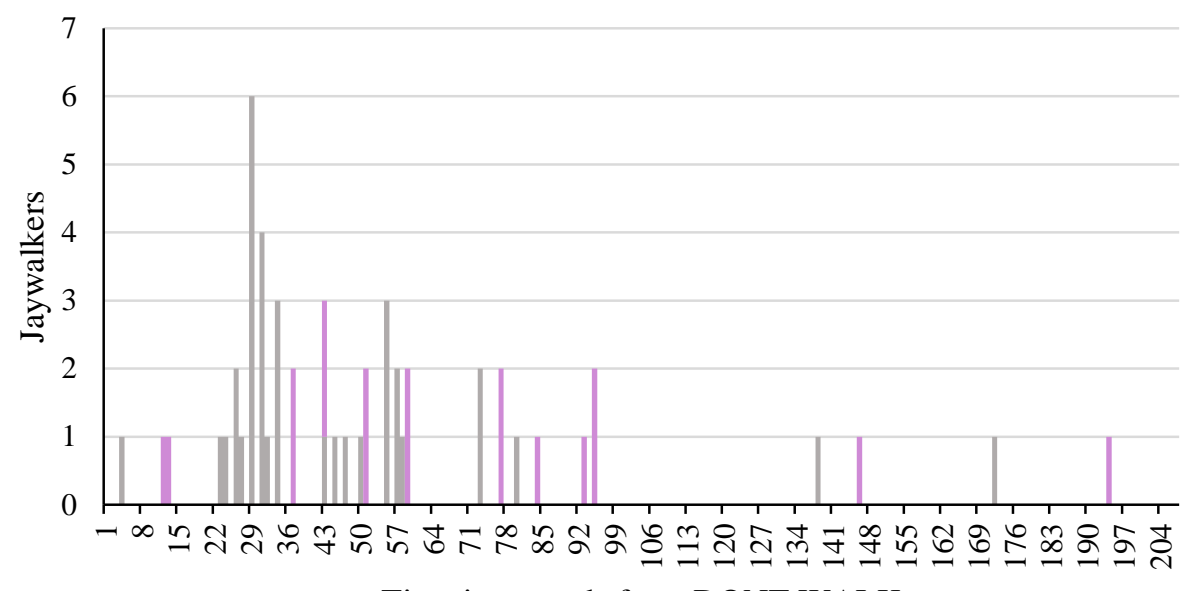

Time in seconds from DONT WALK

• Parallel $\square$ Orthogonal

(a) Peak

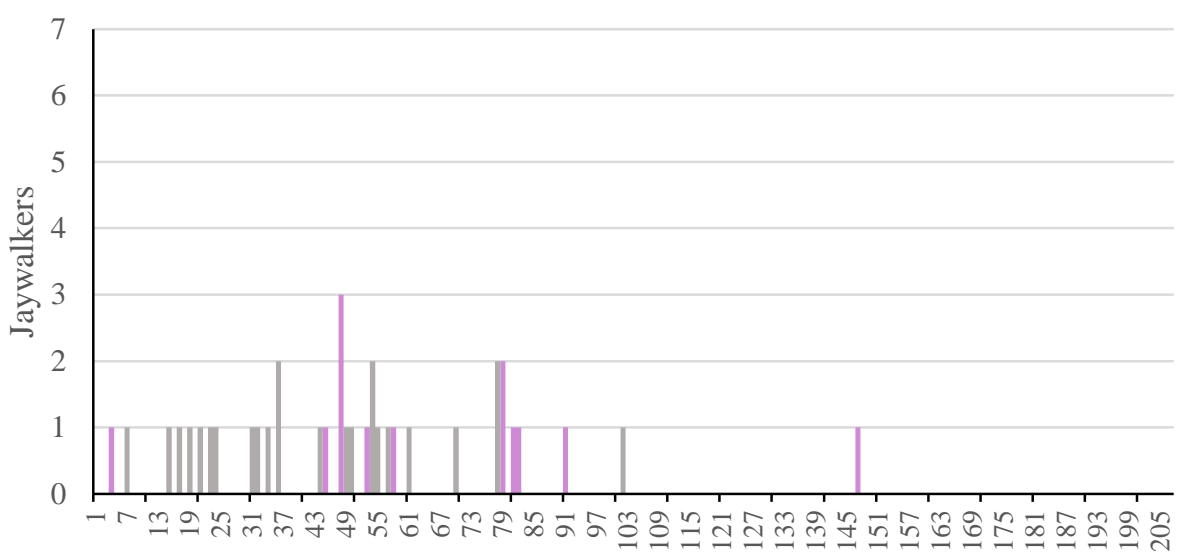

Time in seconds from DONT WALK

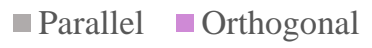

(b) Non-peak

Figure 5.3 Grant Street intersection: Time to jaywalk. 


\subsection{Chauncey Avenue Pedestrian and Vehicle Conflict}

In this study, pedestrian and right turning vehicle conflicts are modeled by using the aggregate conflict, pedestrian, and vehicle occupancies for each cycle. Figure 5.4a shows the occupancy data for one cycle of the analysis period at the Chauncey and State intersection. The $\mathrm{x}$-axis is the time since the beginning of the green phase and the occupancies for the vehicle, pedestrian, and phase status are shown. The periods of time that both the pedestrian and vehicle zone are occupied are highlighted over the conflict areas (callout i). The large, dotted box (callout ii) at the end of the cycle shows a period of 21 seconds, which was calculated by the average time to complete a right turn at this intersection (3.5 seconds) multiplied by the number of right turning vehicles for this phase (6). This time value can be used to adjust the amount of walk time to withhold to allow right-turning vehicles to move through the intersection. Figure 5.4b shows one example of a conflict between a pedestrian (callout iii) and a vehicle (callout iv).

Figure 5.5 displays this information in the form of column charts for 100 cycles in the peak period (Figure 5.5a) and 100 cycles in the non-peak period (Figure 5.5b). The Conflict Occupancy Ratio (COR) is computed by dividing the time when the crosswalk is occupied by a pedestrian during WALK and flashing DON'T WALK (occpedestrian) and a right-turning vehicle is occupying the turning area during phase green (occ vehicle) by the green split $\left(g_{c}\right)$ of a concurrent pedestrian phase:

$$
C O R=\frac{\text { occ }_{\text {pedestrian }} \cap \text { occ }}{\text { vehicle }} \text { Equation } 1
$$

The turning area is the space between the east-side crosswalk and the north-side crosswalk that right turning vehicles drive through to make a right turn. It is the area a right-turning vehicle would wait when a pedestrian is crossing at the crosswalk. In each graph, the cycles are sorted by the COR value cycle to the far left, then by the cycles with the highest occ $_{\text {pedestrian, }}$, then by the cycles with highest occ $_{\text {vehicle }}$. In the peak period, the highest COR is at $24 \%$, shaded by the red bars (callout i), with the overall green occupied for that split at 59\%. In the non-peak period, the highest COR is at $7 \%$, with the overall green occupied for that split at $25 \%$. There is identified opportunities to make use of the extra $41 \%$ and $75 \%$ unoccupied time for the busiest cycles during the peak and non-peak periods to reduce the number of vehicle-pedestrian conflicts.

The 200 cycles in this study are calculated by manually reviewing the footage of each cycle and documenting the occupancy. In the future, the COR can be estimated using the methodology proposed in this study by an automated algorithm that tracks pedestrian and vehicle movements. Automating this performance measure can scale conflict identification for practitioners and operators who monitor intersections daily, or to evaluate intersection performance measures. 


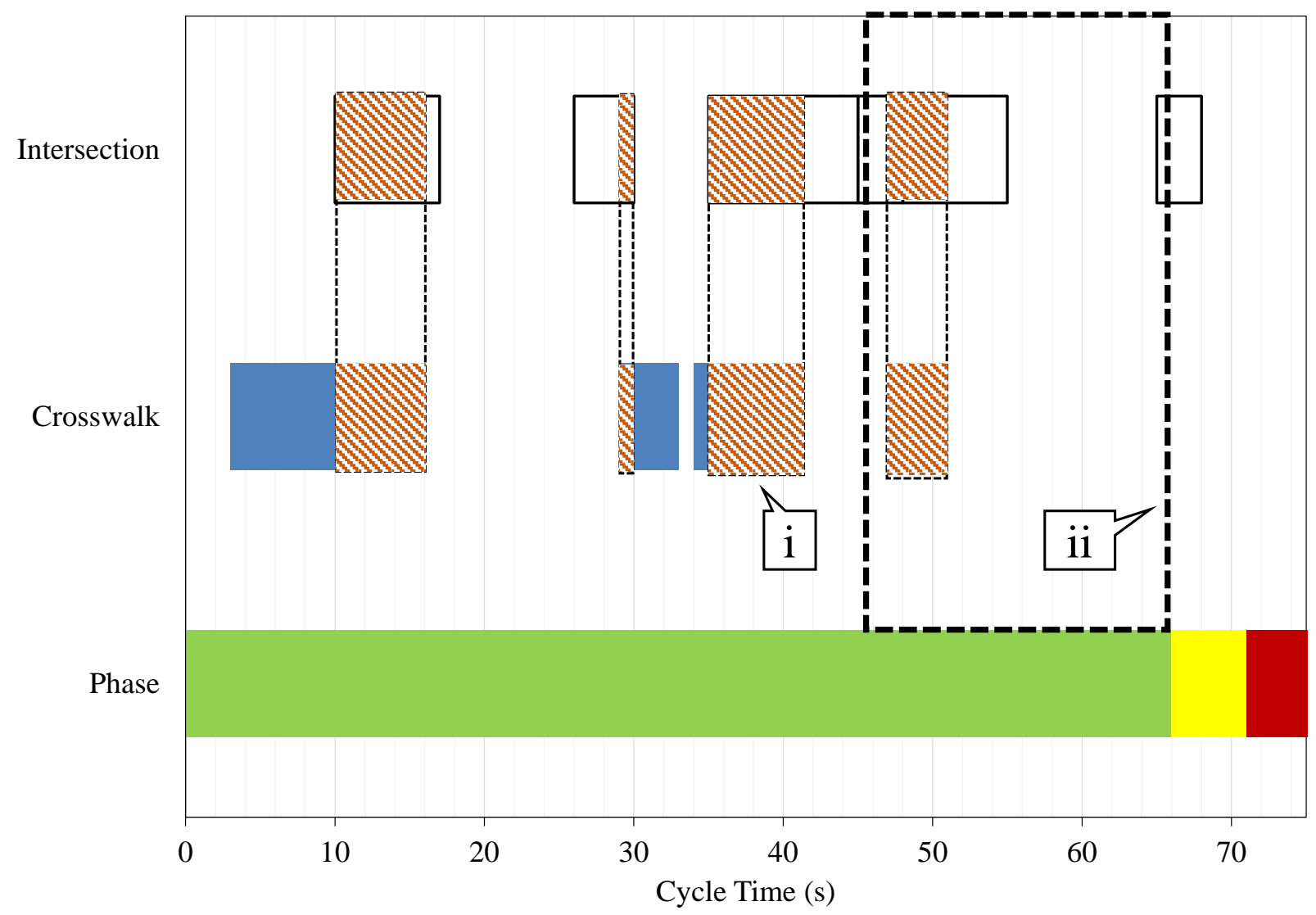

(a) Occupancy chart

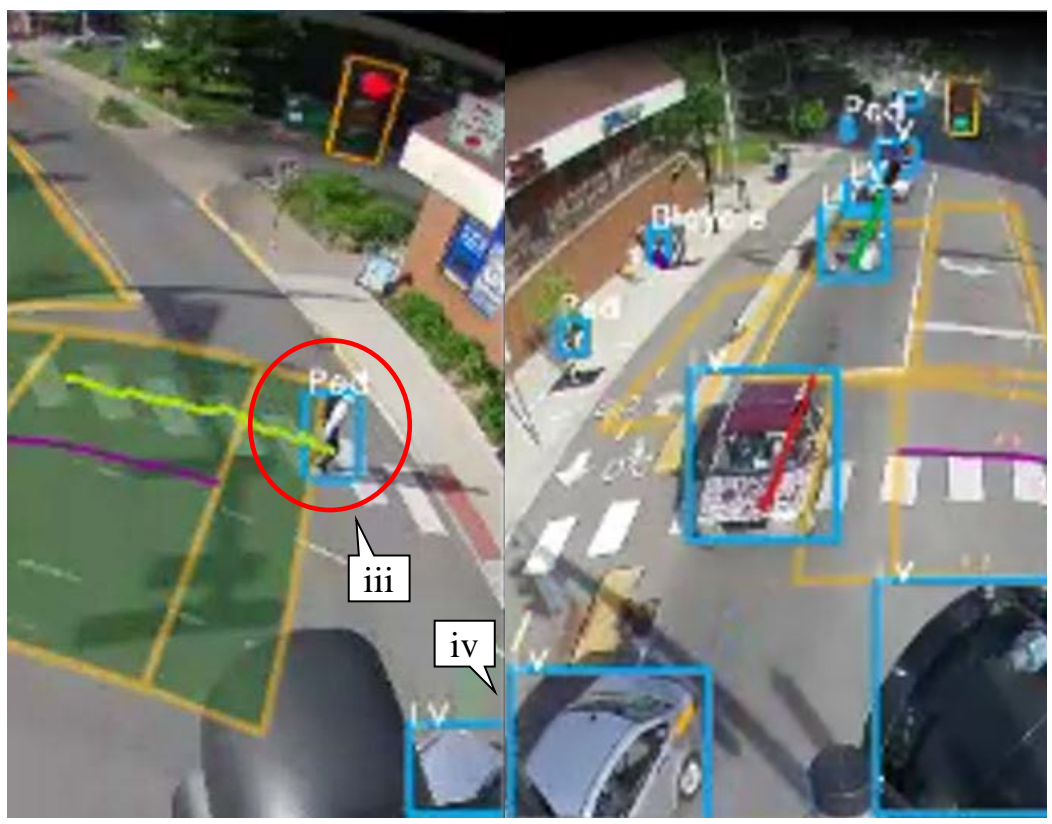

(b) Conflict scenario (callout i)

Figure 5.4 Chauncey Avenue: Occupancy of vehicles and pedestrians relative to the signal phasing. 


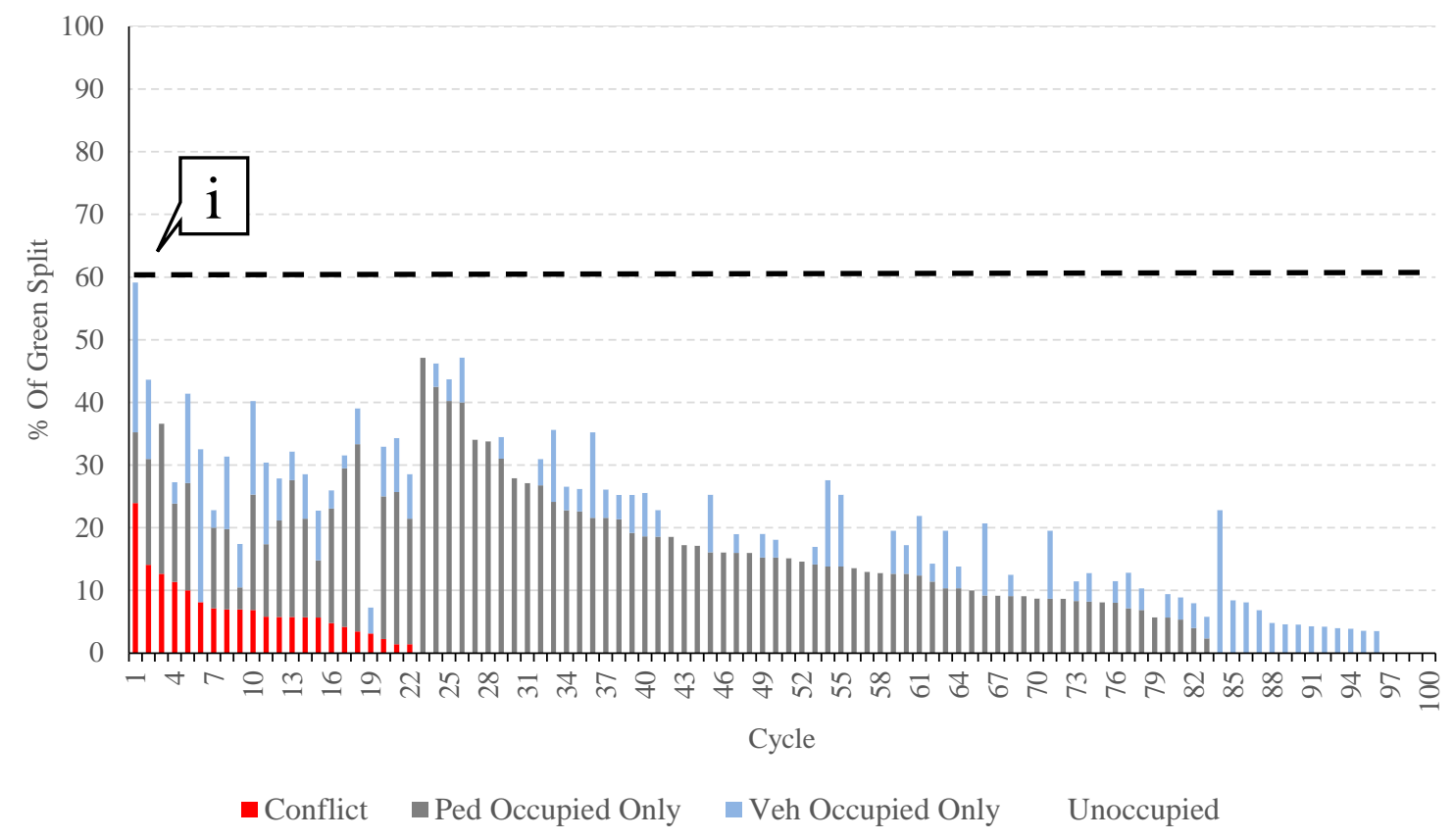

(a) Peak periods

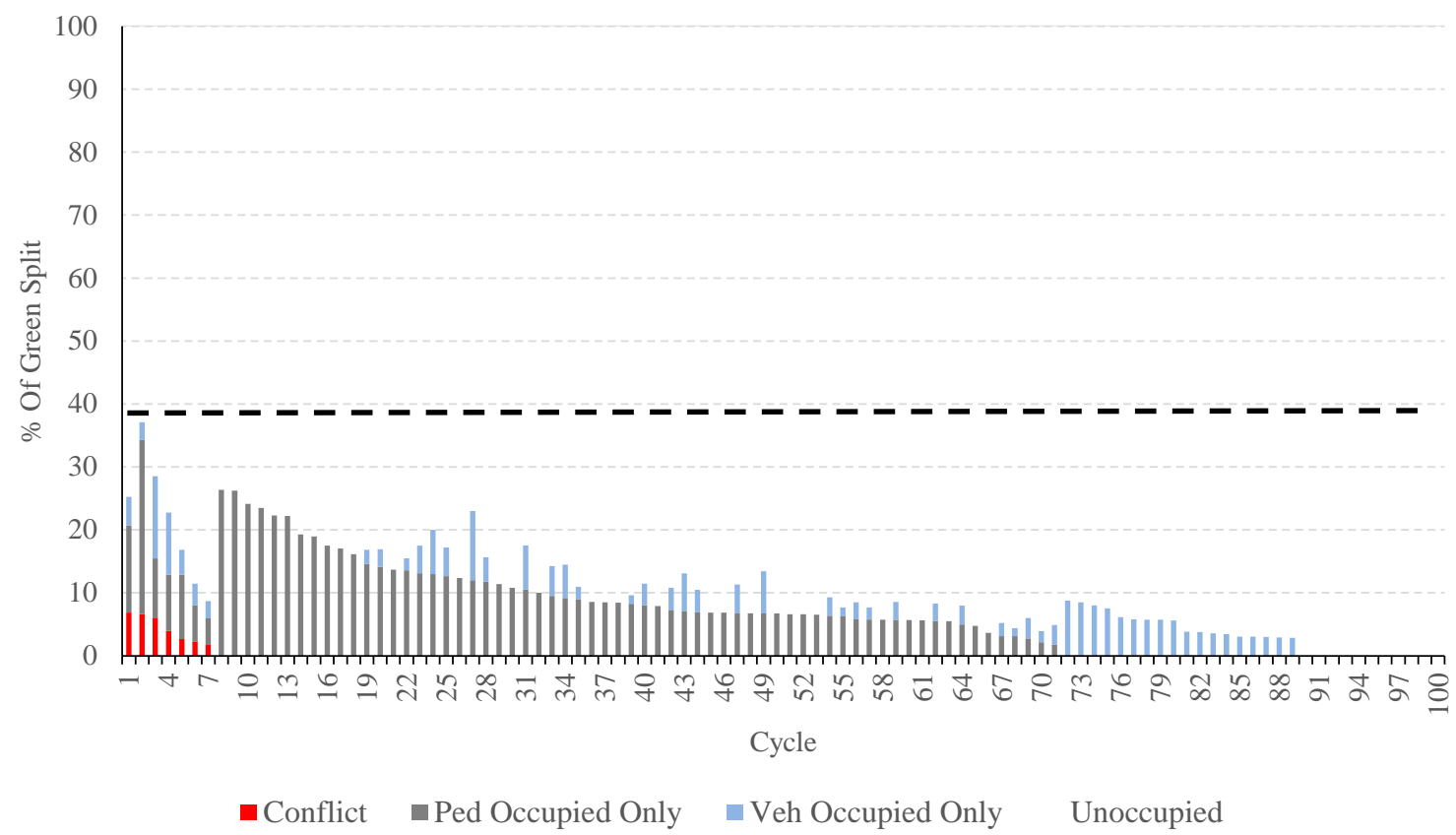

(b) Non-peak periods

Figure 5.5 Chauncey Avenue: Pedestrian and vehicle occupancy sorted by COR. 


\section{SUMMARY}

This study assessed pedestrian detection capabilities of a video detection system using the video recordings at two intersections with pedestrian and vehicle movements. The following two new performance measures were developed from reviewing the footage:

1. The time-to-jaywalk of a pedestrian relative to the start of the solid DON'T WALK.

2. The Conflict Occupancy Ratio (COR), describing the percentage of a concurrent vehicular green phase and pedestrian WALK and flashing DON'T WALK interval where pedestrian-vehicle conflicts were present at a crosswalk.

The median time for a jaywalking event after the onset of solid DON'T WALK was 42 seconds in the peak period and 36 seconds in the non-peak period. The median dwell time of a pedestrian before deciding to jaywalk was 16 seconds in the peak period and 8 seconds in the non-peak period.

A total of 200 cycles were reviewed at one intersection for pedestrian and right turning vehicle conflicts. Of these 200, pedestrians were identified in $154(77 \%)$ of the cycles with a COR ranging from $7 \%$ in the off-peak to $24 \%$ in the peak period. Out of 200 cycles, $62 \%$ of the cycles had pedestrians only and $15 \%$ had vehicles only, with $6.5 \%$ of cycles unoccupied. The highest percentage of occupancy for pedestrians was $47 \%$ and for vehicles was $24 \%$ in any of the observed cycles. Estimating the number conflicts and rate of jaywalking at scale could be extremely useful to practitioners. The COR can also help to characterize the pedestrian and vehicular interactions at different intersections to determine the worst cycles in a time-of-day period.

The pedestrian identification system was accurate within 2 pedestrians per cycle for low volume periods. This analysis was done by examining when the visual graphic display indicated pedestrians. The data was sufficient for calculating the pedestrian performance measures as a screening tool for further analysis. Based upon these results, it is recommended the vendor provide tabular data for the pedestrian activity that can be used to directly compute jaywalking rate and COR. Future anticipated improvements to video detection algorithms and pedestrian/micro-mobility detection systems will only increase the accuracy of these performance measures. 


\section{ACKNOWLEDGEMENTS}

The authors would like to thank the City of West Lafayette for providing access to the study intersections, hardware and software, and Miovision Technologies, Inc. for system support. The Miovision TrafficLink Multimodal Detection System was used to record the intersections in this paper. The contents of this paper reflect the views of the authors, who are responsible for the facts and the accuracy of the data presented herein, and do not necessarily reflect the official views or policies of the sponsoring organizations. These contents do not constitute a standard, specification, or regulation.

\section{AUTHOR CONTRIBUTION}

The authors confirm contribution to the paper as follows:

(a) Study conception and design: Howell Li, Jack Bell, Ben Anderson

(b) Data collection: Jack Bell, Rik Law, Ben Anderson

(c) Analysis and interpretation of results: Jack Bell, Rik Law, Howell Li

(d) Draft manuscript preparation: Jack Bell, Rik Law, Howell Li, Darcy Bullock, Ben Anderson

All authors reviewed the results and approved the final version of the manuscript. 


\section{REFERENCES}

1. Jouneau, E., and C. Carincotte. Particle-Based Tracking Model for Automatic Anomaly Detection. Proceedings-International Conference on Image Processing, ICIP, 2011, pp. 513-516. https://doi.org/10.1109/ICIP.2011.6116394.

2. NHTSA. Fatality Analysis Reporting System (FARS). National Highway Traffic Safety Administration. https://www-fars.nhtsa.dot.gov/Main/index.aspx. Accessed July 30, 2019.

3. Zheng, Y., R. T. Chase, L. Elefteriadou, B. J. Schroeder, V. P. Sisiopiku, and T. R. Board. Where Do Pedestrians Jaywalk and How to Drivers React?-A Study in a Campus Environment. Transportation Research Board, 94th Annual Meeting, December, 2015.

4. Shurbutt, J. Where Pedestrians Cross the Roadway. Publication FHWA-HRT-13-099. FHWA. U.S. Department of Transportation, 2013.

5. Holland, C., and R. Hill. The Effect of Age, Gender and Driver Status on Pedestrians' Intentions to Cross the Road in Risky Situations. Accident Analysis and Prevention, Vol. 39, No. 2, 2007, pp. 224-237. https://doi.org/10.1016/j.aap.2006.07.003.

6. Zhou, R., W. J. Horrey, and R. Yu. The Effect of Conformity Tendency on Pedestrians' Road-Crossing Intentions in China: An Application of the Theory of Planned Behavior. Accident Analysis and Prevention, Vol. 41, No. 3, 2009, pp. 491-497. https://doi.org/10.1016/j.aap.2009.01.007.

7. PEDSAFE. Pedestrian Signal Timing. Pedestrian Safety Guide and Countermeasure Selection System. http://pedbikesafe.org/PEDSAFE/countermeasures_detail.cfm?CM_NUM=47. Accessed July 29, 2019.

8. Zhang, Y., S. A. Mamun, J. N. Ivan, N. Ravishanker, and K. Haque. Safety Effects of Exclusive and Concurrent Signal Phasing for Pedestrian Crossing. Accident Analysis and Prevention, Vol. 83, 2015, pp. 26-36. https://doi.org/10.1016/j.aap.2015.06.010.

9. Hubbard, S. M. L., D. M. Bullock, and C. M. Day. Integration of Real-Time Pedestrian Performance Measures into Existing Infrastructure of Traffic Signal System. Transportation Research Record: Journal of the Transportation Research Board, Vol. 2080, No. 1, 2009, pp. 37-47. https://doi.org/10.3141/2080-05.

10. Ji, Y., J. Hu, L. Li, F. Wang, Y. Su, and D. Yao. Majority-Game Based Conflict Modeling for Pedestrians and Right-Turning Vehicles in Signalized Intersection. IEEE Intelligent Vehicles Symposium Proceedings, 2008, pp. 1191-1196. https://doi.org/10.1109/IVS.2008.4621128.

11. Akin, D., and V. P. Sisiopiku. Pedestrian crossing compliance characteristics at-grade signalized crosswalks: Casestudy in a downtown-university campus environment. 86th Annual Meeting of Transportation Research Board, Vol. 5355, No. 101, 2007, pp. 21-27.

12. Zhao, Y., Y. Bai, and X. Yang. Modeling Conflicts between Right-Turn Vehicles and Pedestrians at Signalized Intersection. ICTIS 2011, pp. 529-536. https://doi.org/10.1061/41177(415)67.

13. Shiravi, S., S. Pidzamecky, and R. Kluger. High Resolution Intersection Pedestrian Performance Measures Using AI Video Detection. No. 1, pp. 1-12.

14. Noyce, D., and B. Bentzen. Determination of Pedestrian Push-Button Activation Duration at Typical Signalized Intersections. Transportation Research Record: Journal of the Transportation Research Board, Vol. 1939, 2007, pp. 63-68.

https://doi.org/10.3141/1939-08. 
15. Federal Highway Administration. Manual on Uniform Traffic Control Devices. 2003, pp. 760.

16. Miovision. Pedestrian and Bicycle Studies.

https://help.miovision.com/s/article/Pedestrian-and-Bicycle-studies--Junction?language=en_US. Accessed Aug. 1, 2019. 